\title{
COMPARISON OF FILTERS DEDICATED TO SPECKLE SUPPRESSION IN SAR IMAGES
}

\author{
P. Kupidura \\ Faculty of Geodesy and Cartography, Warsaw University of Technology, pl. Politechniki 1, 00-661, Warsaw, Poland - \\ p.kupidura@gik.pw.edu.pl
}

Commission VII, WG VII/4

KEY WORDS: SAR Images, Radar Images, Filtering, Mathematical Morphology, Speckle Suppression

\begin{abstract}
:
This paper presents the results of research on the effectiveness of different filtering methods dedicated to speckle suppression in SAR images. The tests were performed on RadarSat-2 images and on an artificial image treated with simulated speckle noise. The research analysed the performance of particular filters related to the effectiveness of speckle suppression and to the ability to preserve image details and edges. Speckle is a phenomenon inherent to radar images - a deterministic noise connected with land cover type, but also causing significant changes in digital numbers of pixels. As a result, it may affect interpretation, classification and other processes concerning radar images. Speckle, resembling "salt and pepper" noise, has the form of a set of relatively small groups of pixels of values markedly different from values of other pixels representing the same type of land cover. Suppression of this noise may also cause suppression of small image details, therefore the ability to preserve the important parts of an image, was analysed as well. In the present study, selected filters were tested, and methods dedicated particularly to speckle noise suppression: Frost, Gamma-MAP, Lee, Lee-Sigma, Local Region, general filtering methods which might be effective in this respect: Mean, Median, in addition to morphological filters (alternate sequential filters with multiple structuring element and by reconstruction). The analysis presented in this paper compared the effectiveness of different filtering methods. It proved that some of the dedicated radar filters are efficient tools for speckle suppression, but also demonstrated a significant efficiency of the morphological approach, especially its ability to preserve image details.
\end{abstract}

\section{INTRODUCTION}

SAR images, as a type of coherent imagery, are inherently affected by speckle. Speckle is a deterministic effect, depending on the scattering of the surface, which can be used to help distinguish certain land use/cover classes (Xia, Sheng, 1996; Kupidura et al., 2010; Wołyńska, 2015). It takes the form of granular noise reducing the quality of the image and, despite its deterministic nature, due to its complexity it must be treated using statistical methods. In the recent years a significant number of speckle suppression filters were developed. A number of studies were dedicated to this issue (e.g. Huan, van Genderen, 1996; Sheng, Xia, 1996; Mansourpour et al., 2006; Kupidura, Koza, 2008; Argenti et al., 2013). They are based mostly on real SAR images, using different indices such as the Speckle Suppression Index or the Feature Preserving Index (Sheng, Xia, 1996) to analyse selected characteristics of the tested filters. In addition to the tests carried out on SAR images, to help evaluate the performance of selected filters quantitatively, the present study also uses artificial images treated with simulated speckle noise (assuming its Gaussian distribution), based on referential speckle-free images. The present study explored the filters included in the Erdas IMAGINE Radar Module software, but also selected morphological filters whose high efficiency in the suppression of impulse noise was suggested in previous research (Kupidura, 2006; Kupidura, Koza, 2008; Kupidura, Jakubiak, 2009; Kupidura et al., 2010). Morphological filters were processed using BlueNote open-source software.

\section{BRIEF REVIEW OF SPECKLE SUPPRESSION FILTERS}

This section offers a brief review of filters tested in this study, dedicated to speckle suppression from single polarisation SAR images. The filters are grouped in three sections, depending on their construction and purpose.

\subsection{Simple filters}

Filters presented in this section are simplistic operations which may be used to remove or suppress speckle in SAR images.

Mean filter: The pixel is replaced by the average of all values within the filter window. It does not remove the speckle from the image, but averages it into one. Theoretically, dark and bright speckle pixels within the filter window can cancel each other out. The probability of such situations increases with the size of the filter window. However, it produces image blur, loss of details and, eventually, loss of spatial resolution.

Median filter: The pixel of interest is replaced be the median value within the filter window. This filter is a useful tool for removing pulse noise (such as speckle) - noise of less than a half of the filter window could be suppressed or even removed, not blurred, as in the case of the mean filter. The median filter is edge preserving (Pratt, 1991), although it may lead to the removing (or suppressing) of small (also linear) objects from the image, exactly in the same way as it removes (or suppresses) speckle noise. 


\subsection{Complex filters}

The filters presented below are more complex and dedicated particularly to radar images and speckle suppression.

Local Region: It divides the filter window into eight regions and calculates the variance for each of them. Then the pixel of interest is replaced by the mean value of the most homogenous region (i.e. with the lowest variance) - assumed to be less affected by the speckle noise (Nagao, Matsuyama, 1979).

Frost filter: This filter is derived from the minimum mean square error algorithm. Its kernel adapts to the local statistical values of the image, so it can suppress the speckle while at the same preserving the edges (Frost et al., 1982).

Lee filter: The Lee filter is reportedly the first model-based filter dedicated to speckle noise suppression (Argenti et al., 2013). It is also derived from the minimum mean square error algorithm (Lee, 1980, 1981).

Lee-sigma filter: This filter is based on the Gaussian distribution model. It averages only the pixels within a certain standard deviation range.

Gamma MAP filter: It is based on the assumption that the original value of the degraded pixel lies between the local average and its actual value. The MAP (maximum a posteriori) filter assumes Gamma distribution for speckle noise (and not Gaussian like Lee, Lee-Sigma or Frost filters). (Lopes et al., 1990)

\subsection{Morphological alternate filters}

The filters discussed in this section are based on morphological operations: opening and closing. Sequentially used, these operations have the ability to remove or suppress dark (opening) and bright (closing) speckle (Serra, 1982; Vincent, 1992; Nieniewski, 1998). These are alternate filters: CO (closing followed by opening) and OC (opening then closing). They vary depending on the type of operations (simple, with multiple structuring element and/or by reconstruction). Despite two different forms of alternate filters ( $\mathrm{CO}$ and $\mathrm{OC}$ ), only one of them $(\mathrm{OC})$ is presented in the results below, as no significant difference between them was observed. Also, for the size of the structuring element (SE; an equivalent of filter window in other types of filters) over 1 , it is recommended to apply a combination of filters of sequentially increasing SE for a better preservation of the image structure (Sternberg, 1986; Serra, 1988). Filters of this kind were used in the present study.

Simple alternate filters (SAF): They are based on simple opening and closing in their original form as presented by Serra (1982) or Haralick et al. (1987). They are effective operations for removing impulse noise, but they also strongly affect the structure of the filtered image, removing or changing the objects in it.

Filters with multiple structuring element (MSE): These filters are based on a modified closing and opening operation. Such a modification was presented by Song and Delp (1990), and Cheng and Venetsanopoulos (1992), and it uses a combination of multiple images obtained with a single SE, together composing a multiple SE. This kind of filter may be edge preserving (Cheng and Venetsanopoulos, 1992; Kupidura, 2006; Kupidura, Jakubiak, 2009) or area preserving (Vincent,
1993), depending on the set of the structuring elements. In the present study, edge preserving filters with MSE were used.

Filters by reconstruction: These filters base on opening and closing by reconstruction, first presented by Soille (1999), based on geodesic reconstruction introduced by Serra (1982). They allow reconstructing objects not completely removed to their original shapes. The principles of geodesic reconstruction might also be applied to operations with MSE. This kind of filters: with MSE by reconstruction, was tested in the study presented below.

\section{EXPERIMENTAL RESULTS}

The efficacy of the selected filters was tested on both the original SAR image (RadarSat-2) and also on artificial images treated with simulated speckle noise.

\subsection{Artificial images}

Artificial images were created to simulate the speckle noise and allow assessing the effectiveness of the filters being tested by comparing the filtered images with the original ones before noise introduction. Two images were created: the homogenous one, in order to compare despeckle attributes of the selected filters, and the detailed one, in order to allow the assessment of their ability to preserve image details. The filters were tested on two versions of the detailed image: the original one, and the one treated with simulated noise. The original image was used to allow extracting the influence of the filters on image details only. The speckle was simulated by adding salt and pepper noise processed with a Gaussian filter with filter window size 2, to ensure the Gaussian distribution of the noise.

The effectiveness of the filtration was assessed by comparing the filtration results with the original, speckle-free images. In order to evaluate the performance of the filters quantitatively, the results of the filtering were compared with the speckle-free images. The mean value of the differential images was the main evaluation factor. Also, the standard deviation values of the differential images were calculated to help analyse the results.

The first test image (380x404 pixels) used for numerical experimentation presenting a homogenous area and treated with a simulated speckle is shown in Figure 1.
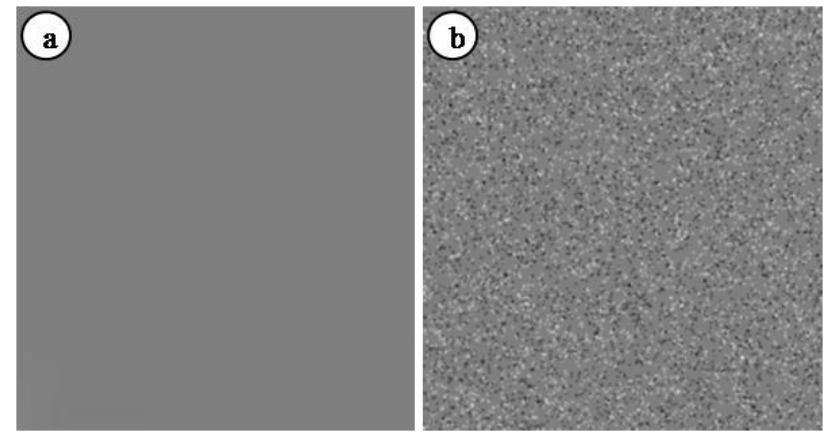

Figure 1. An artificial homogeneous test image; a) reference speckle free image, b) image with a simulated speckle 
Table 1 shows the results of the comparison of filtering results to the speckle-free image (Figure 1a). Figure 2 shows the resulting images of the selected filters.

\begin{tabular}{|c|c|c|c|c|c|c|c|}
\hline $\begin{array}{l}\text { filter } \\
\text { type }\end{array}$ & size & $\begin{array}{c}\text { mean } \\
\text { dif. }\end{array}$ & $\begin{array}{c}\text { dif. } \\
\text { sd }\end{array}$ & $\begin{array}{l}\text { filter } \\
\text { type }\end{array}$ & size & $\begin{array}{c}\text { mean } \\
\text { dif. }\end{array}$ & $\begin{array}{c}\text { dif. } \\
\text { sd }\end{array}$ \\
\hline \multirow{5}{*}{ Mean } & 1 & 8.4 & 8.6 & \multirow{5}{*}{$\begin{array}{l}\text { Lee- } \\
\text { Sigma }\end{array}$} & 1 & 8.4 & 8,6 \\
\hline & 2 & 6.7 & 5.7 & & 2 & 6.8 & 5,9 \\
\hline & 3 & 5.3 & 4.3 & & 3 & 5.4 & 4,5 \\
\hline & 4 & 4.4 & 3.4 & & 4 & 4.5 & 3,7 \\
\hline & 5 & 3.7 & 2.9 & & 5 & 3.8 & 3,2 \\
\hline \multirow{5}{*}{ Median } & 1 & 7.1 & 9.0 & \multirow{5}{*}{$\begin{array}{c}\text { Gamma } \\
\text { MAP }\end{array}$} & 1 & 8.6 & 8,6 \\
\hline & 2 & 4.3 & 5.9 & & 2 & 7.0 & 5,9 \\
\hline & 3 & 2.6 & 3.8 & & 3 & 5.6 & 4,4 \\
\hline & 4 & 1.6 & 2.7 & & 4 & 4.7 & 3,6 \\
\hline & 5 & 1.1 & 2.0 & & 5 & 4.0 & 3,0 \\
\hline \multirow{5}{*}{$\begin{array}{l}\text { Local } \\
\text { Region }\end{array}$} & 1 & 8.2 & 10.8 & \multirow{5}{*}{ SAF } & 1 & 6.3 & 7,3 \\
\hline & 2 & 6.3 & 8.4 & & 2 & 3.0 & 3,9 \\
\hline & 3 & 5.1 & 6.0 & & 3 & 1.9 & 2,8 \\
\hline & 4 & 4.6 & 4.8 & & 4 & 1.2 & 2,2 \\
\hline & 5 & 4.4 & 4.1 & & 5 & 1.0 & 1,8 \\
\hline \multirow{5}{*}{ Frost } & 1 & 8.7 & 9.1 & \multirow{5}{*}{ MSE } & 1 & 8.9 & 10,6 \\
\hline & 2 & 7.3 & 6.6 & & 2 & 7.3 & 8,8 \\
\hline & 3 & 5.9 & 5.0 & & 3 & 5.5 & 7,1 \\
\hline & 4 & 4.8 & 3.9 & & 4 & 3.3 & 4,8 \\
\hline & 5 & 4.0 & 3.2 & & 5 & 2.3 & 3,7 \\
\hline \multirow{5}{*}{ Lee } & 1 & 8.6 & 8.8 & \multirow{5}{*}{$\begin{array}{l}\text { MSE } \\
\text { by } \\
\text { recon. }\end{array}$} & 1 & 9.0 & 10,7 \\
\hline & 2 & 7.1 & 6.5 & & 2 & 8.0 & 9,4 \\
\hline & 3 & 5.9 & 5.6 & & 3 & 6.3 & 7,9 \\
\hline & 4 & 5.1 & 5.4 & & 4 & 4.2 & 5,7 \\
\hline & 5 & 4.7 & 5.5 & & 5 & 3.3 & 4,6 \\
\hline \multicolumn{5}{|c|}{ No filter } & - & 9.8 & 12.1 \\
\hline
\end{tabular}

Table 1. Comparison of results of selected filtration of the

simulated speckled homogenous image with a noise-free reference image; mean dif. is the mean value of the differential image, and dif. $s d$ is its standard deviation value
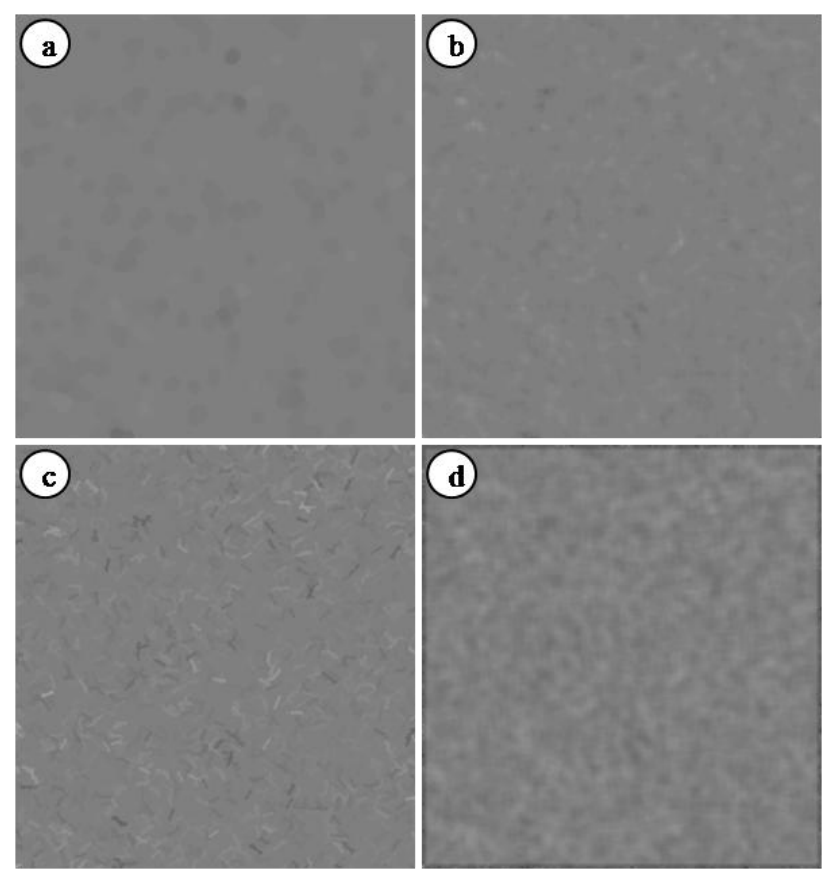

Figure 2. Results of the filtration of homogenous image with simulated speckle, using: a) SAF, b) Median filter, c) MSE filter, d) Lee filter (filter window size: 5)
As expected, the efficiency of all the filters for the homogeneous area increases with the size of the filter window (SE in the case of morphological filters), which corresponds to the size of the simulated speckle. Two filters with the best effectiveness in this case are: the morphological Simple Alternate Filter (Figure 2a) and the Median filter (Figure 2b). Comparatively good results were obtained using the MSE filter (Figure 2c), but the amount of noise left in the image was roughly twice as high as in the case of Median and SAF filters. Among the filters with the biggest filter window size, the worst results were obtained using the Lee filter (Figure 2d), but also in other cases (Local Region, Frost, Gamma MAP) the results were mostly similar. It is worth pointing out that, according to the statistics shown in Table 1, all the filters improved the quality of the image.

The next test image is presented in Figure 3. It is a detailed noise-free image (380x202 pixels).

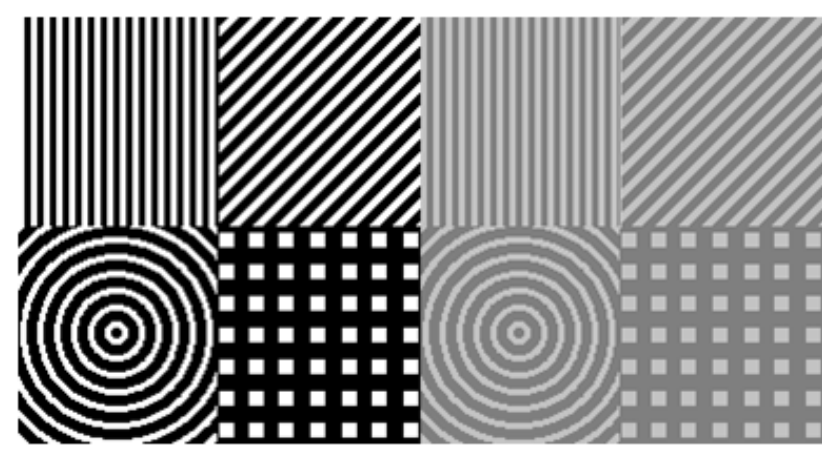

Figure 3. An artificial detailed speckle-free test image

\begin{tabular}{|c|c|c|c|c|c|c|c|}
\hline $\begin{array}{c}\text { filter } \\
\text { type }\end{array}$ & size & $\begin{array}{c}\text { mean } \\
\text { dif. }\end{array}$ & $\begin{array}{c}\text { dif. } \\
\text { sd }\end{array}$ & $\begin{array}{c}\text { filter } \\
\text { type }\end{array}$ & size & $\begin{array}{c}\text { mean } \\
\text { dif. }\end{array}$ & $\begin{array}{c}\text { dif. } \\
\text { sd }\end{array}$ \\
\hline \multirow{5}{*}{ Mean } & 1 & 26.4 & 33.7 & & 1 & 13.1 & 22.9 \\
& 2 & 46.0 & 39.2 & & 2 & 17.1 & 22.7 \\
& 3 & 63.7 & 49.0 & Lee- & 3 & 20.9 & 23.7 \\
& 4 & 71.9 & 52.1 & Sigma & 4 & 22.6 & 24.0 \\
& 5 & 74.0 & 51.6 & & 5 & 23.1 & 24.0 \\
\hline \multirow{5}{*}{ Median } & 1 & 1.7 & 18.0 & & 1 & 12.8 & 19.1 \\
& 2 & 4.7 & 31.0 & Gamma & 2 & 20.7 & 22.4 \\
& 3 & 55.7 & 94.2 & MAP & 3 & 23.7 & 21.5 \\
& 4 & 76.1 & 103 & & 25.5 & 20.6 \\
& 5 & 81.0 & 104 & & 5 & 15.2 & 20.7 \\
\hline \multirow{5}{*}{ Local } & 1 & 21.8 & 55.4 & & 1 & 4.5 & 30.4 \\
Region & 2 & 35.8 & 67.4 & & 2 & 52.3 & 92.0 \\
& 3 & 59.7 & 78.8 & SAF & 3 & 55.7 & 94.0 \\
& 4 & 69.7 & 76.1 & & 4 & 63.4 & 97.7 \\
& 5 & 70.6 & 69.7 & & 5 & 63.4 & 97.7 \\
\hline \multirow{5}{*}{ Frost } & 1 & 3.0 & 5.6 & & 1 & 0.0 & 0.4 \\
& 2 & 6.2 & 8.5 & & 2 & 0.0 & 0.4 \\
& 3 & 8.8 & 10.9 & MSE & 3 & 0.0 & 2.8 \\
& 4 & 10.8 & 12.6 & & 4 & 0.9 & 14.1 \\
& 5 & 12.1 & 13.9 & & 5 & 10.2 & 45.9 \\
\hline \multirow{5}{*}{ Lee } & 1 & 54.8 & 87.4 & & 1 & 0.0 & 0.4 \\
& 2 & 40.2 & 54.6 & MSE & 2 & 0.0 & 0.4 \\
& 3 & 43.5 & 40.0 & by & 3 & 0.0 & 0.6 \\
& 4 & 38.7 & 22.0 & recon. & 4 & 0.1 & 4.4 \\
& 5 & 39.0 & 19.5 & & 5 & 10.2 & 45.8 \\
\hline
\end{tabular}

Table 2. Comparison of the results of selected filtration of a simulated speckle-free detailed image to the source reference image; mean dif. is the mean value of the differential image, and dif. $s d$ is its standard deviation value 
The results presented in Table 2 show the differences between the filtered image and the original image itself (Figure 3). It allows assessing the detail and edge preservation characteristics of the tested filters. Figure 4 shows the results of selected operations.

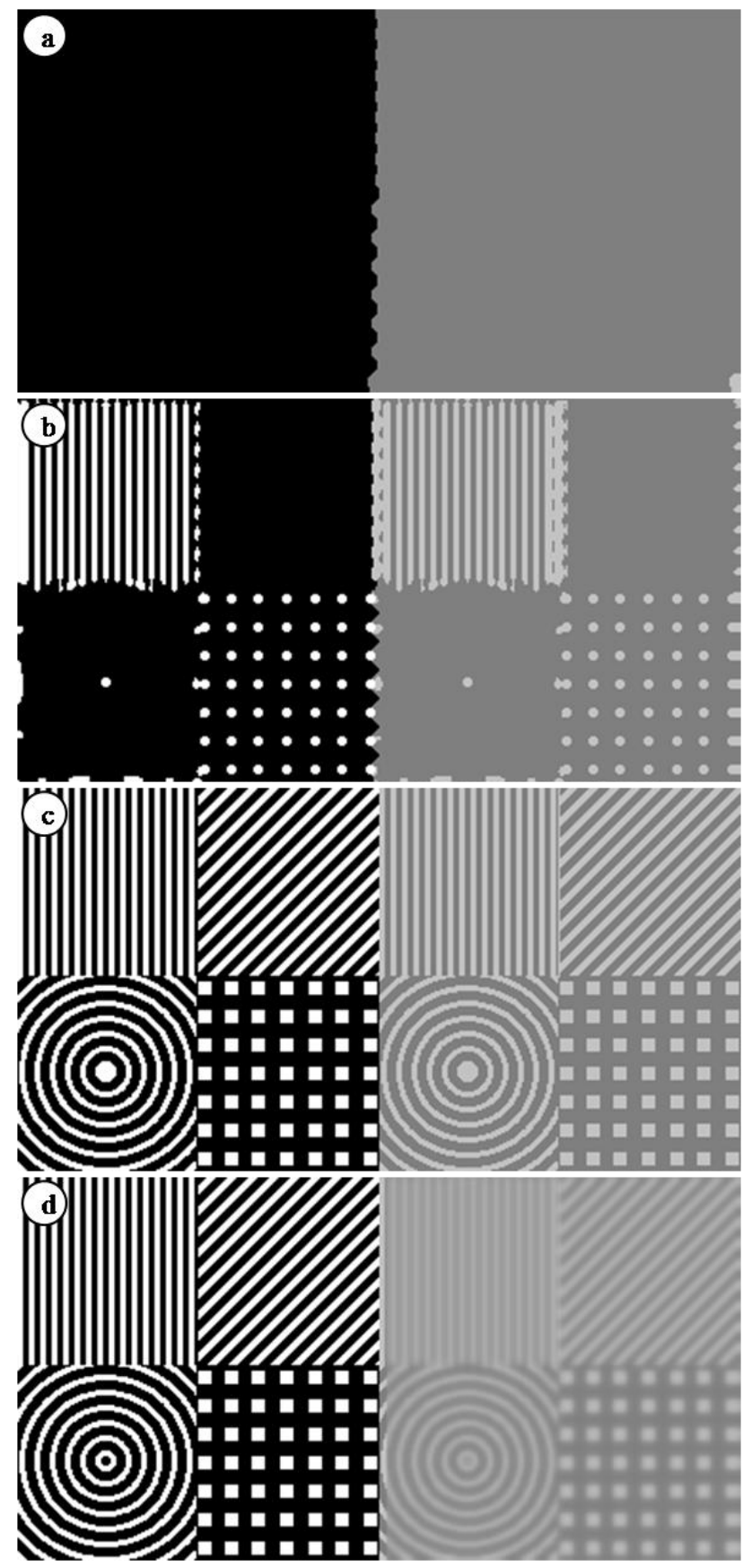

Figure 4. Results of the filtration of detailed speckle-free image using: a) SAF, b) Median filter, c) MSE filter by reconstruction,

d) Frost filter; filter window (SE) size: 4

As expected, this ability decreases with the strength of the filter, i.e. size of the filter window (or SE) (with the exception of the Lee filter). Also, SAF (Figure 4a) and Median filter (Figure 4b) show the worst (together with the Mean filter) detail-preserving ability, particularly their versions using bigger filter window (or $\mathrm{SE}$ ). Unequivocally, the best results were obtained using the MSE and MSE by reconstruction (Figure 4c) filters. For SE sizes $1-4$, the detail preservation of these filters is close to perfection. Only for the biggest test size of structuring element -5 , the differences between the filtration results and the original image were found to be significant, but still much smaller than for the other filters. Relatively good detail preservation abilities were also demonstrated by the Frost filter (Figure 4d), though were considerably worse than those of MSE filters, due especially to the blurring effect imposed on the less contrasted parts of the image (high contrast details are very well preserved).

The last of the simulated test images is the detailed image (380x202 pixels), presented above in Figure 3, but affected with simulated speckle (Figure 5). Table 3 presents the results of filtration of the image from Figure 5. Figure 6 shows the result images of the selected filters.

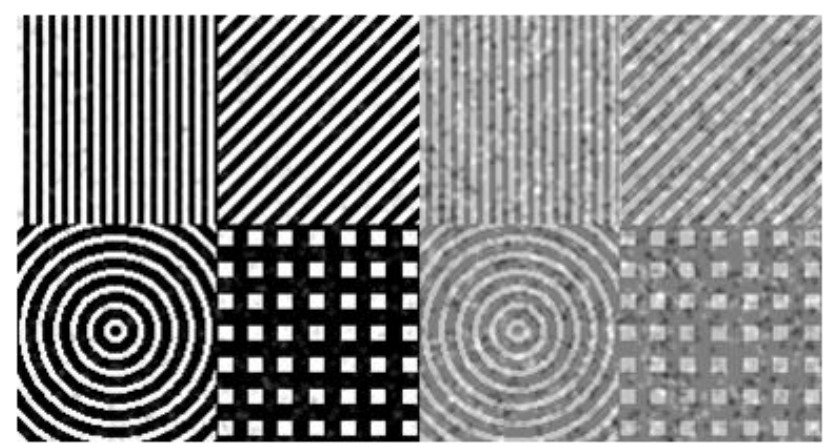

Figure 5. An artificial detailed test image affected with simulated speckle

Surprisingly, almost none of the tested filters showed more similarity to the original (not-speckled) image than the unfiltered image. This is caused by the relatively significant modifications induced in the high-contrasted objects in the image by the filters, particularly the effect of edge blurring. The best results were again produced by the MSE (Figure 6a) and MSE by reconstruction (Figure 6b) filters. Only these filters (with SE size 1-4) showed more similarity to the original image than the unfiltered image, which proves their good abilities in both speckle suppression and detail preservation. The second best speckle suppression with good detail-preserving abilities was achieved with the Frost filter (Figure 6c), particularly due to its satisfying edge-preserving capacity. Gamma-MAP (Figure 6d) and Lee-Sigma filters produced relatively good results, but significantly worse than the best morphological filters, particularly regarding the modifications they made in the details of the filtered image. However, it is worth pointing out that Frost, Gamma-Map and Lee filters preserve high contrast details very well and the decrease of the quality depends on the blurring effect visible on the low-contrast details of the image (Figures 6c and 6d). The best speckle suppressors: the Median filter and the morphological Simple Alternate Filter (SAF), also strongly modified the image itself, so their general results were among the worst within the tested group of filters. 


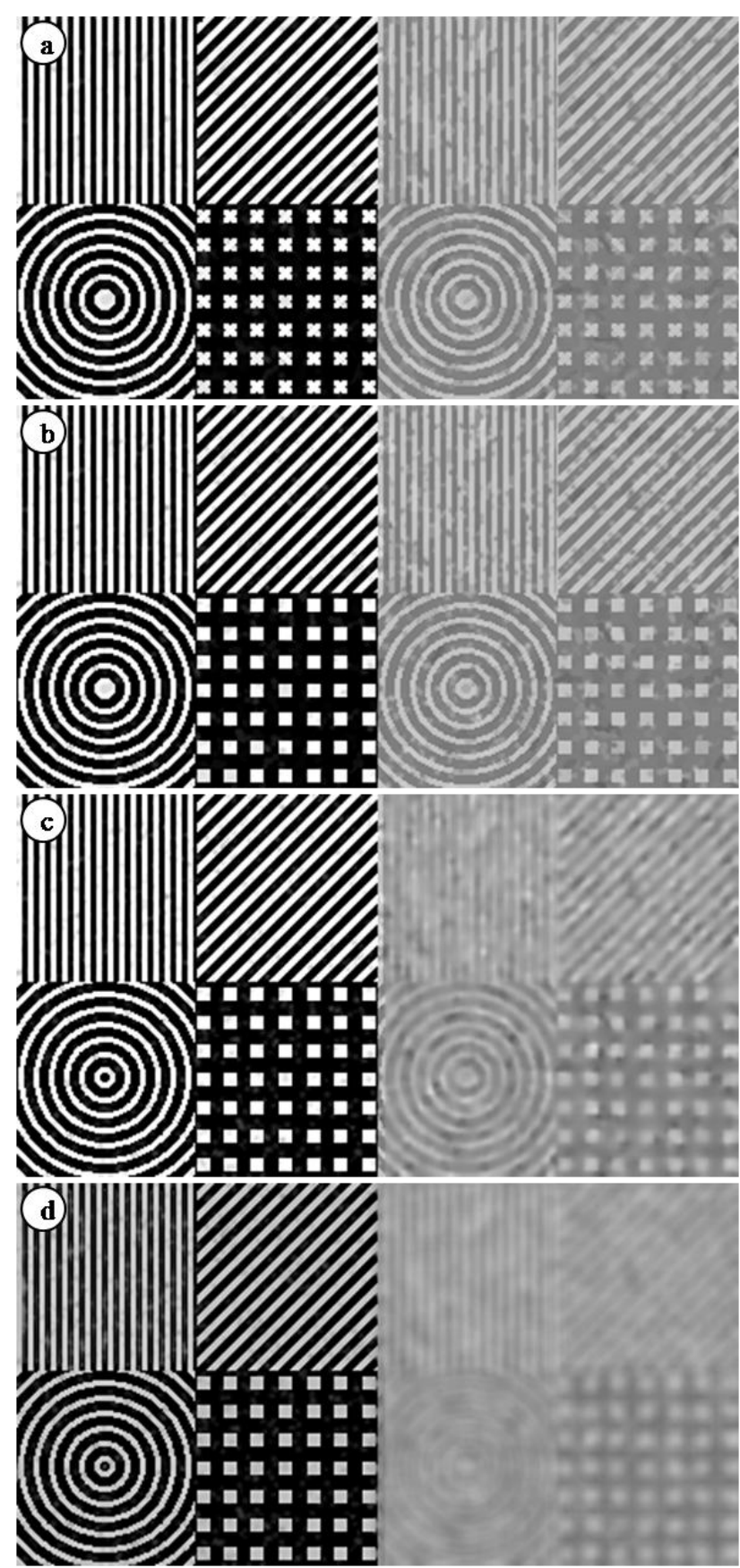

Figure 6. Results of the filtration of a detailed image with simulated speckle, using: a) MSE filter, b) MSE filter by reconstruction, c) Frost, d) Gamma-MAP filter; filter window (SE) size: 4

\begin{tabular}{|c|c|c|c|c|c|c|c|}
\hline $\begin{array}{l}\text { filter } \\
\text { type }\end{array}$ & size & $\begin{array}{c}\text { mean } \\
\text { dif. }\end{array}$ & $\begin{array}{c}\text { dif. } \\
\text { sd }\end{array}$ & $\begin{array}{l}\text { filter } \\
\text { type }\end{array}$ & size & $\begin{array}{c}\text { mean } \\
\text { dif. }\end{array}$ & $\begin{array}{c}\text { dif. } \\
\text { sd }\end{array}$ \\
\hline \multirow{5}{*}{ Mean } & 1 & 30.1 & 33.1 & \multirow{5}{*}{$\begin{array}{l}\text { Lee- } \\
\text { Sigma }\end{array}$} & 1 & 15.8 & 20.6 \\
\hline & 2 & 47.7 & 39.2 & & 2 & 18.2 & 20.5 \\
\hline & 3 & 64.4 & 48.6 & & 3 & 21.2 & 21.3 \\
\hline & 4 & 72.2 & 51.8 & & 4 & 22.4 & 21.5 \\
\hline & 5 & 74.2 & 51.3 & & 5 & 22.7 & 21.3 \\
\hline \multirow{5}{*}{ Median } & 1 & 8.1 & 19.4 & \multirow{5}{*}{$\begin{array}{c}\text { Gamma } \\
\text { MAP }\end{array}$} & 1 & 17.3 & 20.2 \\
\hline & 2 & 11.7 & 29.9 & & 2 & 23.7 & 23.6 \\
\hline & 3 & 56.3 & 86.1 & & 3 & 26.8 & 22.8 \\
\hline & 4 & 74.2 & 94.7 & & 4 & 28.8 & 22.4 \\
\hline & 5 & 78.3 & 95.1 & & 5 & 28.1 & 21.8 \\
\hline \multirow{5}{*}{$\begin{array}{l}\text { Local } \\
\text { Region }\end{array}$} & 1 & 25.6 & 51.8 & \multirow{5}{*}{ SAF } & 1 & 11.3 & 29.8 \\
\hline & 2 & 39.6 & 65.3 & & 2 & 53.4 & 87.7 \\
\hline & 3 & 60.2 & 75.4 & & 3 & 57.1 & 90.4 \\
\hline & 4 & 69.1 & 72.7 & & 4 & 63.5 & 95.2 \\
\hline & 5 & 71.7 & 68.0 & & 5 & 63.6 & 95.5 \\
\hline \multirow{5}{*}{ Frost } & 1 & 8,4 & 10,6 & \multirow{5}{*}{ MSE } & 1 & 6,8 & 10,4 \\
\hline & 2 & 9,8 & 11,4 & & 2 & 6,2 & 9,8 \\
\hline & 3 & 11,3 & 12,4 & & 3 & 5,8 & 9,6 \\
\hline & 4 & 12,8 & 13,3 & & 4 & 6,6 & 15,3 \\
\hline & 5 & 13,9 & 13,9 & & 5 & 14,6 & 42,0 \\
\hline \multirow{5}{*}{ Lee } & 1 & 35,0 & 58,5 & \multirow{5}{*}{$\begin{array}{l}\text { MSE } \\
\text { by } \\
\text { recon. }\end{array}$} & 1 & 6,9 & 10,5 \\
\hline & 2 & 35,0 & 58,5 & & 2 & 6,5 & 10,0 \\
\hline & 3 & 38,3 & 25,4 & & 3 & 5,9 & 9,6 \\
\hline & 4 & 41,3 & 23,8 & & 4 & 5,6 & 10,0 \\
\hline & 5 & 51,5 & 21,5 & & 5 & 13,7 & 40,5 \\
\hline \multicolumn{5}{|c|}{ No filter } & - & 7,3 & 11,2 \\
\hline
\end{tabular}

Table 3. Comparison of results of selected filtration of a simulated speckled detailed image to a noise-free reference image; mean dif. is the mean value of the differential image, and dif. $s d$ is its standard deviation value

\subsection{RadarSat2}

For the purpose of the analysis, two fragments of Radarsat-2 imagery (single polarisation HV) were used. The first one presents water, a part of the image with high homogeneity (Figure 7). This image was used to analyse the capacity for speckle suppression.

The analysis of images presented in Figure 7 (original image in Figure 7a) confirms the results obtained during the tests of the simulated image. The best speckle suppression capacity was found in morphological Simple Alternate Filter and Median filters; the results of these filtrations show the highest homogeneity of the presented area. Morphological filters with MSE (respectively, Figures $7 \mathrm{f}$ and $7 \mathrm{~b}$ ) have relatively good speckle suppression properties. The water surface is more homogenous after filtration with the use of these filters. The differences between other filters are relatively small, but a higher "smoothness" of the MSE filter (Figure 7g) can be noticed, confirming good efficacy of this filter in this respect. 

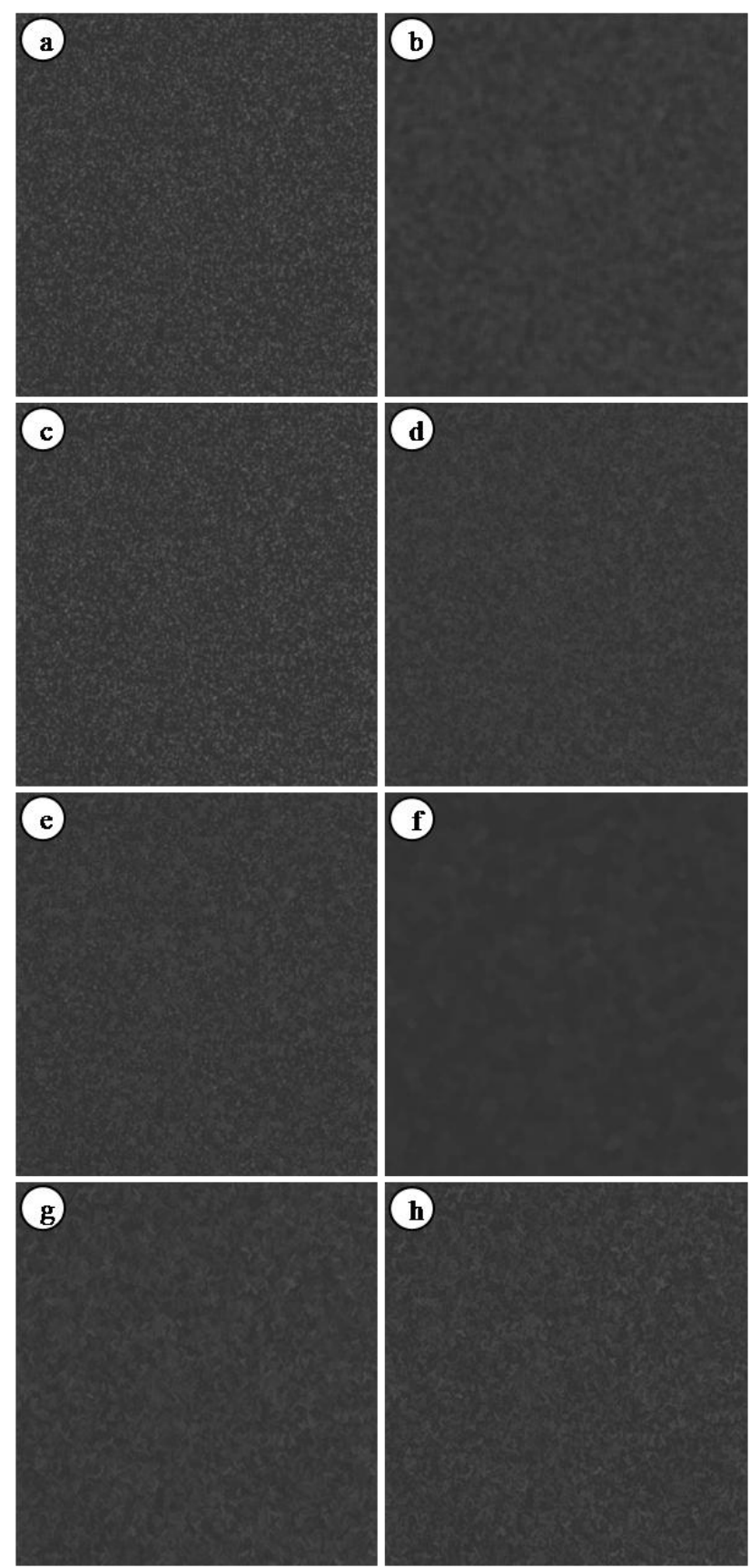

Figure 7. A homogenous water area; a) the original Radarsat-2 image and the results of its filtration, using: b) Median filter, c) Frost filter, d) Lee-sigma filter, e) Gamma-MAP filter, f) SAF, g) MSE filter, h) MSE filter by reconstruction; filter window

\section{(SE) size: 3}

Table 4 presents the standard deviation values of the images after filtration using selected operations. No classic Speckle Suppression Index (Sheng, Xia, 1996), using a decreasing of standard deviation as well as the similarity of mean values of source and result images, was evaluated, because water surface, as generally dark in SAR imagery, is affected mostly by the light speckle, so the successful filtration must also lead to the decrease of the mean value. In this case, only a decrease of standard deviation, assessing the increase of homogeneity of the image, was evaluated.
The results confirm the analysis performed on the test images: the Simple Alternate Filter shows very good capabilities for speckle suppression, better than the Mean and Median filters. The MSE filter presents a satisfying performance, compared to classic adaptive filters; also, the homogeneity of its results is considerably higher than the homogeneity of the results of MSE filtration by reconstruction.

\begin{tabular}{|c|c|c|c|c|c|}
\hline $\begin{array}{c}\text { filter } \\
\text { type }\end{array}$ & size & sd & $\begin{array}{c}\text { filter } \\
\text { type }\end{array}$ & size & sd \\
\hline \multirow{5}{*}{ Mean } & 1 & 3.38 & & 1 & 3,38 \\
& 2 & 1.94 & Lee- & 2 & 2,93 \\
& 3 & 1.50 & Sigma & 3 & 2,75 \\
& 4 & 1.26 & 4 & 2,66 \\
& 5 & 1.10 & & 5 & 2,62 \\
\hline & 1 & 3.37 & & 1 & 3,63 \\
& 2 & 2.43 & Gamma & 2 & 3,47 \\
Median & 3 & 1.94 & MAP & 3 & 3,46 \\
& 4 & 1.63 & & 3,47 \\
& 5 & 1.43 & & 5 & 3,47 \\
\hline & 1 & 4.01 & & 1 & 2,12 \\
Local & 2 & 2.80 & & 2 & 1,17 \\
Region & 3 & 2.10 & SAF & 3 & 0,90 \\
& 4 & 1.73 & & 4 & 0,72 \\
& 5 & 1.49 & & 5 & 0,65 \\
\hline \multirow{5}{*}{ Frost } & 1 & 4.83 & & 1 & 3,95 \\
& 2 & 4.84 & & 2 & 3,46 \\
& 3 & 4.84 & MSE & 3 & 2,81 \\
& 4 & 4.84 & & 4 & 2,13 \\
& 5 & 4.84 & & 5 & 1,75 \\
\hline \multirow{5}{*}{ Lee } & 1 & 18.9 & & 1 & 4,11 \\
& 2 & 3.08 & MSE & 2 & 3,78 \\
& 3 & 2.73 & by & 3 & 3,34 \\
& 4 & 2.73 & recon. & 4 & 2,76 \\
& 5 & 2.69 & & 5 & 2,45 \\
\hline & No filter & & - & 4.84 \\
\hline
\end{tabular}

Table 4. Standard deviation values of the images after filtration using selected filters

The second fragment of the Radarsat-2 image shows a detailed image of the Vancouver urban area (Figure 8). Its purpose is to analyse the capacity of selected filters for preserving details and edges.

In this case, the best speckle suppression filters, i.e. SAF (Figure 8f) and Median filters (Figure 8b), demonstrate rather poor efficiency: the details are mostly removed from the image. Lee-Sigma (Figure 8d) and Gamma-MAP (Figure 8e) show relatively good detail preserving abilities, but some of the details, especially smaller ones, are also removed or blurred. Also most of the edges of the objects in the images are blurred. The Frost filter (Figure 8c) presents the best properties of this kind, but considering the mediocre efficiency of speckle suppression, it seems it can be attributed to the strength of the filter with selected parameters. MSE filters (Figures $8 \mathrm{~g}$ and $8 \mathrm{~h}$ ) also preserve details, particularly those of linear type, and are edge preserving. 

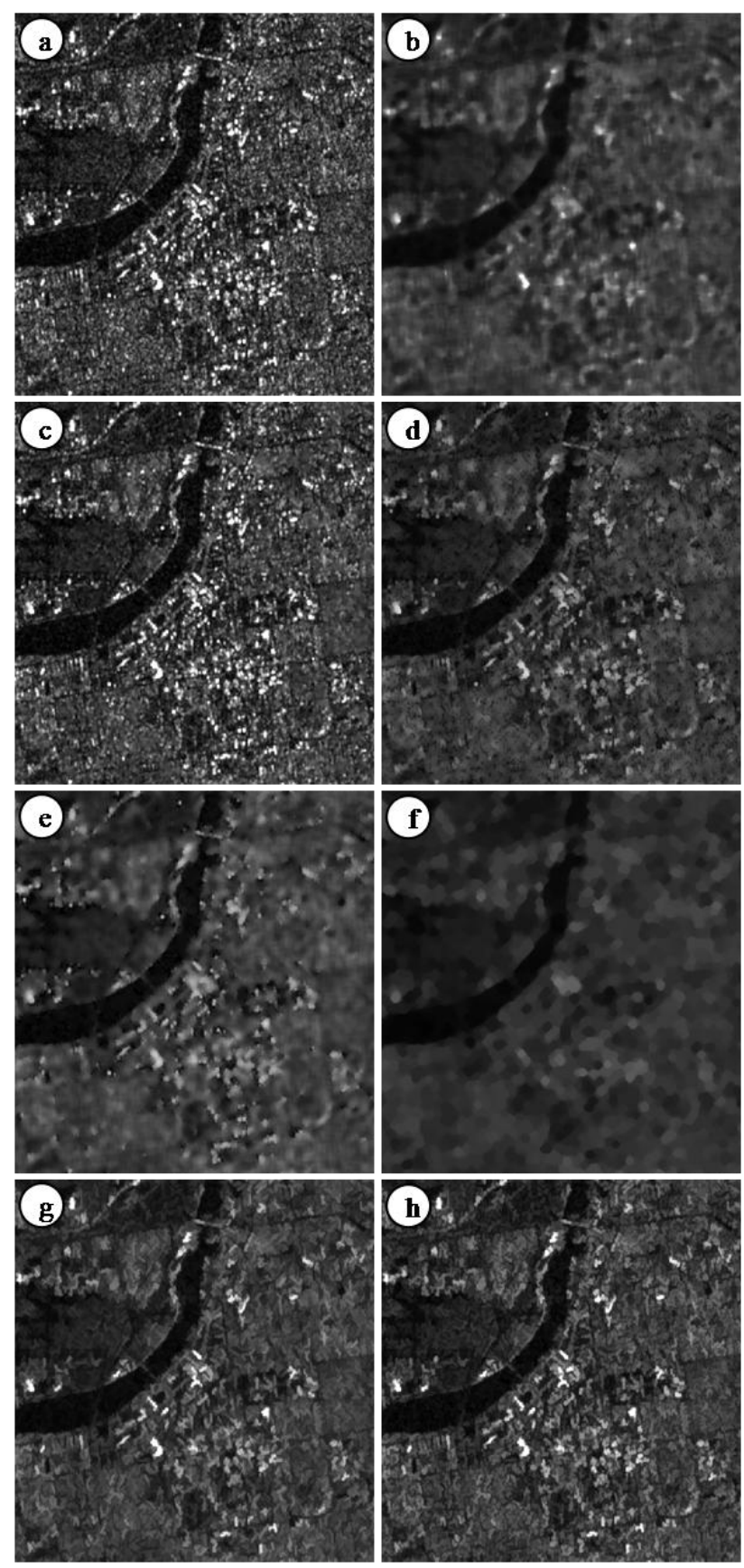

Figure 8. A detailed image of an urban area; a) the original

Radarsat-2 image and the results of its filtration, using: b) Median filter, c) Frost filter, d) Lee-sigma filter, e) Gamma-

MAP filter, f) SAF, g) MSE filter, h) MSE filter by reconstruction; size of mask (SE) for all filters: 3

\section{CONCLUSIONS}

This paper reviewed the effect of applying 10 selected filters on a simulated image as well as real SAR (Radarsat-2) imagery. The research corroborated the results of the previous studies on the efficacy of non-morphological speckle filters, i.e. edge and detail-preserving performance of the Frost filter and poor performance of Mean, Median and Local Region filters in this respect.
The research also proved a promising potential of morphological filters. The Simple Alternate Filter, based on simple morphological operations, performed poorly on a detailed image, but it demonstrated a strong capacity for speckle suppression. The performance of two other morphological filters: with multiple structuring element (MSE) and with multiple structuring element by reconstruction, was very effective: they are detail-preserving but also suppress speckle considerably well: similarly or even better than other adaptive filters such as Frost, Gamma-Map or Lee.

Morphological filters are different in nature than the other tested filters. They are not adaptive in the strict sense of the word. In contrast to such filters as Lee, Frost or Gamma-MAP, the principle of operation is based on the geometric properties of the image (size and shape of the objects), not on the statistics of pixel values. In the case of speckle - impulse noise, it produces very good results, although objects of smaller size can also be removed, depending on the size of the structuring element.

The obtained results point out that the use of adaptive rules, derived for example from the Frost filter, would potentially improve the detail-preserving properties of morphological filters with multiple structuring element, and therefore their efficacy.

\section{REFERENCES}

Argenti, F., Lapini, A., Bianchi, T., Alparone, L. 2013. A Tutorial on Speckle Reduction in Synthetic Aperture Radar Images. IEEE Geoscience And Remote Sensing Magazine, 1(3), pp. 6-35.

Cheng, F., Venetsanopoulos, A.N. 1992. An Adaptive Morphological Filter for Image Processing. IEEE Transactions on Image Processing, 1(4), 533-539.

Frost, V.S., Stiles, J.A., Shanmugan K.S., Holtzman, J.C. 1982. Amodel for radar images and its application to adaptive digital filtering of multiplicative noise. IEEE Transactions on Pattern Analysis and Machine Intelligence, vol. PAMI-4, no. 2, pp. 157-166.

Goodman, J.W. 1976. Some fundamental properties of speckle, The Journal of the Optical Society of America, 66(11), pp. $1145-1150$

Haralick, R.M., Sternberg, S.R., Zhuang, X. 1987. Image Analysis using Mathematical Morphology. IEEE Transactions on Pattern Analysis and Machine Intelligence, 9(4), pp. 532550 .

Huang, Y., van Genderen, J.L., 1996. Evaluation of Several Filtering Techniques for ERS-1\&2 Imagery. International Archives of Photogrammetry and Remote Sensing XXXI, B2, pp. 164-169.

Kupidura, P. 2006. Zastosowanie wybranych operacji morfologii matematycznej do wydzielania klas pokrycia terenu na zdjęciach satelitarnych. PhD thesis, Warsaw University of Technology, Warsaw.

Kupidura, P., Jakubiak, M. 2009. The morphological filtering of the remote sensing images for the noise reduction comparing to traditional filters. Annals of Geomatics, VII 2(32), pp. 63-68.

Kupidura, P., Koza, P. 2008. Radar imagery filtering with use of the mathematical morphology operations. Polish Journal of Environmental Studies, 17 (1C), pp. 31-35. 
Kupidura, P., Koza, P., Marciniak, J. 2010. Morfologia matematyczna $w$ teledetekcji. Wydawnictwo Naukowe PWN, Warsaw, pp. 250.

Lee, J.S. 1980. Digital image enhancement and noise filtering by use of local statistics. IEEE Transactions on Pattern Analysis and Machine Intelligence, vol. PAMI-2, no. 2, pp. $165-168$.

Lee, J.S. 1981. Speckle Analysis and Smoothing of Synthetic Aperture Radar Images. Computer Graphics and Image Processing, 17 (1), pp. 24-32.

Lopes, A., Nezry, E., Touzi, R., Laur, H. 1990. Maximum A Posteriori Speckle Filtering and First Order Textural Models in SAR Images. Paper presented at the International Geoscience and Remote Sensing Symposium (IGARSS), College Park, Maryland, pp. 2409-2412.

Mansourpour, M., Rajabi, M.A., Blais, J.A.R. 2006. Effects and Performance of Speckle Noise Reduction Filters on Active Radars and SAR Images. International Archives of ISPRS, XXXVI 1/W41, Ankara

Nagao, M., Matsuyama, T., 1979. Edge preserving smoothing. Computer Graphics and Image Processing, 9(4), pp. 394-407.

Nieniewski, M. 1998. Morfologia matematyczna w przetwarzaniu obrazów. Akademicka Oficyna Wydawnicza PLJ, Warsaw.

Pratt, W. K. 1991. Digital Image Processing. 2nd ed. New York: John Wiley \& Sons, Inc.

Serra, J. 1982. Image Analysis and Mathematical Morphology, Vol. 1. Academic Press, London.

Serra, J. (ed.). 1988. Image Analysis and Mathematical Morphology, Vol. 2: Theoretical Advances. Academic Press, London, 1988.

Sheng, Y., Xia, Z.G., 1996. A Comprehensive Evaluation of Filters for Radar Speckle Suppression. IEEE International Geoscience and Remote Sensing Symposium Proceedings, 3, pp. 1559-1561.

Soille, P. 1999. Morphological Image Analysis. Springer Verlag, Berlin.

Song, J., Delp, E.J. 1990. The analysis of morphological filters with multiple structuring elements. Computer Vision Graphics and Image Processing, 50, pp. 308-328.

Sternberg, S.R. 1986. Grayscale Morphology. Computer Vision Graphics and Image Processing, 35(3), pp. 333-355.

Vincent, L. 1993. Grayscale Area Opening and Closing, their efficient implementation and applications. Proceedings of Eurasip Workshop on Mathematical Morphology and its Applications to Signal Processing, Barcelona.

Wołyńska, K. 2015. Wykorzystanie szumu cętkowego do klasyfikacji pokrycia terenu na podstawie obrazów radarowych. Master thesis, Warsaw University of Technology, Warsaw pp. 138.

Xia, Z.G., Sheng, Y. 1996. Radar speckle: noise or information? Geoscience and Remote Sensing Symposium, IGARSS '96, Remote Sensing for a Sustainable Future, Lincoln, 1, pp. 48-50. 